\title{
Avaliação sanitária dos serviços de hemoterapia do Estado do Paraná
}

\author{
Health surveillance of the hemotherapy services of \\ the State of Paraná, Brazil
}

Renata Pavese ${ }^{1}$, Edson Zangiacomi Martinez ${ }^{2}$

1. Secretaria de Saúde do Estado do Paraná, Centro de Hematologia e Hemoterapia do Paraná HEMEPAR, Curitiba, PR, Brasil. E-mail: renata_pavese@hotmail.com. ORCID: 0000-0001-7698-6716

2. Faculdade de Medicina de Ribeirão Preto, Universidade de São Paulo, Ribeirão Preto, SP, Brasil. E-mail: edson@fmrp.usp.br. ORCID: 0000-0002-0949-3222

CONTATO: Edson Zangiacomi Martinez | Faculdade de Medicina de Ribeirão Preto, Universidade de São Paulo | Avenida Bandeirantes 3900 - Vila Monte Alegre | Ribeirão Preto | São Paulo | CEP 14049-900 | Brasil | Telefone: (16) 3602-2569 | E-mail: edson@fmrp.usp.br

COMO CITAR: Pavese R, Martinez EZ. Avaliação sanitária dos serviços de hemoterapia do estado do Paraná. R. Saúde Públ. Paraná. 2020 Jul;3(1):97-107.

COPYRIGHT Esta obra é disponibilizada nos termos da Licença Creative Commons - 4. 0
Internacional. É permitida a reprodução parcial ou total desta obra, desde que citada a fonte.

RESUMO O objetivo deste estudo é avaliar os riscos potenciais apresentados pelos serviços de hemoterapia do estado do Paraná no período de 2010 a 2017, e identificar as não conformidades comuns nas Agências Transfusionais no ano de 2017. Foram utilizados dados do sistema informatizado SHTWEB. Utilizou-se o Método de Avaliação de Risco Potencial em Serviços de Hemoterapia (MARPSH). Em 2010, 2013 e 2017, foram inspecionados respectivamente $57,1 \%, 79,1 \%$ e 59,7\% dos serviços, sendo esta cobertura relativamente baixa, e respectivamente 41,8\%, 51,9\% e $67,2 \%$ dos serviços foram classificados como de baixo risco. Em 2017, as não conformidades mais frequentes relacionaram-se a equipamentos e dispositivos, infraestrutura, gestão da qualidade, recursos humanos e retrovigilância. Em conclusão, são necessárias medidas para a melhoria dos resultados dos serviços de hemoterapia avaliados, incluindo prioridades com relação a sistemas de informação, equipamentos e dispositivos, gestão da qualidade, recursos humanos e hemovigilância, além da necessidade de aumentar a cobertura das inspeções sanitárias. 
PALAVRAS-CHAVE: Vigilância Sanitária. Avaliação de Programas e Projetos de Saúde. Bancos de Sangue. Serviço de Hemoterapia. Gestão de Riscos.

\begin{abstract}
The objective of this study is to evaluate the potential risks presented by the hemotherapy services of the State of Paraná, Brazil, from 2010 to 2017, and to identify common nonconformities in transfusion agencies in 2017. Data from the SHTWEB computer system were used. The Potential Risk Assessment Method in Hemotherapy Services (MARPSH) was used. In 2010, 2013 and 2017, respectively 57.1\%, 79.1\% and 59.7\% of the services were inspected. This coverage was relatively low, and respectively $41.8 \%, 51.9 \%$ and $67.2 \%$ of services were classified as low risk. In 2017, the most frequent nonconformities were related to equipment and devices, infrastructure, quality management, human resources, and retrovigilance. In conclusion, strategies are needed to improve the results of the evaluated hemotherapy services, including priority actions for the information systems, equipment and devices, quality management, human resources and haemovigilance, and the need to increase health inspection coverage.
\end{abstract}

KEYWORDS: Health Surveillance. Program Evaluation. Blood Banks. Hemotherapy Service. Risk Management.

\title{
INTRODUÇÃO
}

A

hemoterapia é uma prática multiprofissional voltada ao tratamento de doenças pela administração de sangue e/ou hemoderivados'. Dado que as transfusões são procedimentos que apresentam riscos de transmissão de doenças infecciosas e eventos adversos, tornam-se necessárias estratégias de seleção adequada de doadores de sangue, uso de testes efetivos para o sangue coletado, a manutenção de um adequado sistema de hemovigilância e legislações e regulamentos técnicos dirigidos aos serviços de produção ${ }^{1-3}$.

Em 1999, através da Lei nº. 9.782, a Agência Nacional de Vigilância Sanitária (ANVISA) criou o Sistema Nacional de Vigilância Sanitária (SNVS). Trata-se de uma autarquia brasileira com finalidade institucional de promover a proteção da saúde da população, por intermédio do controle sanitário da produção e consumo de produtos e serviços submetidos à vigilância sanitária4

Essa lei estabeleceu que o sistema de regulação de sangue no Brasil, entre as diferentes esferas governamentais, deve atuar de forma descentralizada. Para tanto, as esferas dos niveis municipal e estadual devem se organizar para executar as atividades de fiscalização e inspeção sanitária localmente, e reportar-se à ANVISA, que é responsável pela coordenação nacional do SNVS, para a definição de normas regulatórias nacionais e a elaboração de roteiros de inspeção padronizados ${ }^{4.5}$. 
Dada a complexidade organizacional dos serviços de hemoterapia, são necessárias legislações e políticas próprias para a melhoria do processo de todo o ciclo do sangue, incluindo ações que englobam a proteção do doador ao receptor ${ }^{6}$. Entre outros documentos, as ações da vigilância sanitária estão embasadas em legislações que dispõem sobre as Boas Práticas do Ciclo do Sangue (Resolução da Diretoria Colegiada RDC n 34/2014); ; no capítulo II da Portaria de Consolidação n 5, de 28 de setembro de 2017, que consolida as normas sobre as ações e os serviços de saúde do Sistema Único de Saúde ${ }^{8}$; Portaria Conjunta MS/SAS n 370/2014, que dispõe sobre o regulamento técnico sanitário para o transporte de sangue e componentes ${ }^{9}$; a RDC n² 20/2014, que dispõe sobre o regulamento técnico sanitário para o transporte de material biológico humano ${ }^{10}$; e a RDC 151/2001, que aprova o regulamento técnico sobre os níveis de complexidade dos serviços de hemoterapia"1.

De modo geral, as ações de vigilância são compostas por inspeções sanitárias, investigações de eventos e monitoramento de produtos e riscos. As inspeções nos serviços de hemoterapia consistem em avaliações de estrutura física, recursos humanos, procedimentos técnicos e de gestão, processos de trabalho e registros dos serviços de hemoterapia e, para isso, utilizam um roteiro de inspeção instituído pela ANVISA ${ }^{12,13}$, composto por cinco módulos que se referem: o Módulo I - às informações gerais, o Módulo II - à captação, recepção/ registro, triagem clínica e coleta, o Módulo III - à triagem laboratorial, o Módulo IV - ao processamento, rotulagem, armazenamento e distribuição e o Módulo $\vee$ - à agência transfusional, terapia transfusional e outros procedimentos terapêuticos.

Já o Método de Avaliação de Risco Potencial em Serviços de Hemoterapia (MARPSH) é comumente utilizado após a inspeção, para a avaliação de risco da unidade inspecionada'14. Este instrumento surgiu da necessidade de se detectar os riscos potenciais dos serviços avaliados, sendo que a gradativa inserção dos resultados avaliativos dos serviços de hemoterapia provenientes do MARPSH, além de sistematizar o monitoramento das não conformidades observadas nos serviços de hemoterapia, possibilitam a inserção desses dados em boletins e relatórios de atividades da ANVISA, além de evidenciar indicadores de desempenho dos serviços.

Diante do exposto, o presente artigo tem por objetivos avaliar os riscos potenciais apresentados pelos serviços de hemoterapia do estado do Paraná no período de 2010 a 2017, e identificar as não conformidades comuns nas Agências Transfusionais no ano de 2017.

\section{MÉTODO}

Trata-se de um estudo descritivo, baseado no perfil sanitário dos Serviços de Hemoterapia no estado do Paraná. Todos os dados utilizados foram obtidos do Sistema Estadual de Informação e Controle Hemoterápico do Paraná (SHTWEB) ${ }^{15}$, a partir do consentimento formal do Centro Estadual de Vigilância Sanitária (CEVS) da Secretaria da Saúde do Estado do Paraná (SESA-PR). Foram utilizadas as informações disponíveis em julho de 2019, sendo os dados obtidos do SHTWEB na forma de tabelas, baseadas nos roteiros de inspeção sanitária e classificações de riscos potenciais no período de 2010 a 2017.

Em 2017, havia no estado do Paraná 572 Serviços de Hemoterapia, classificados de acordo com sua tipologia: um Hemocentro Coordenador (HEMEPAR), quatro Hemocentros Regionais, 17 Núcleos de Hemoterapia, oito Unidades de Coleta e Transfusão, 104 Agências Transfusionais (AT) e 438 Serviços Transfusionais. Dentre as 104 AT, 30 estão localizadas e pertencem aos Hemocentros Coordenador e Regionais, Núcleos de Hemoterapia e Unidades de Coleta e Transfusão. Tais serviços estão distribuídos 
geograficamente em 22 regionais de saúde, as quais compõem quatro macrorregiões. O Hemocentro Coordenador e os Hemocentros Regionais são de natureza pública. Dos Núcleos de Hemoterapia, nove são públicos e oito privados, totalizando 17 serviços. Com relação às Unidades de Coleta e Transfusão, todas são de natureza pública, e dentre as AT, 18 são públicas, cinco privadas e 51 privadas com convênio SUS.

O MARPSH é baseado em um roteiro de inspeção constituído por 471 itens avaliativos, podendo ser composto por até cinco módulos, de acordo com o tipo de serviço a ser inspecionado ${ }^{16}$. Nas inspeções em AT são empregados somente o Módulo I (informações gerais), parte do módulo IV (processamento, rotulagem. armazenamento e distribuição) e o Módulo V (terapia transfusional). De forma rotineira, um técnico local, ao realizar a inspeção em cada serviço, classifica cada item como "adequado", "inadequado" ou "não se aplica" (esta opção é usada quando o serviço avaliado não realiza aquela determinada atividade).

Tal instrumento apresenta diferentes categorias de classificação de risco, de acordo com o item avaliado. Itens considerados como nível crítico I se referem a elementos de controle que podem ou não interferir na qualidade e segurança do produto, serviços e procedimentos. O nível II confere certo grau de criticidade de risco e interfere na qualidade dos serviços, procedimentos e produtos. O nível III determina exposição ao risco, se não cumprido ou cumprido inadequadamente, conferindo grau crítico de risco na qualidade e segurança dos serviços ofertados. Para cada nível de criticidade são atribuídos pesos como valores para análise qualitativa e quantitativa. Para o Nivel I é considerado peso 1, para itens com criticidade II é atribuído peso 3 e para itens críticos nível III é atribuído peso 5. A aplicação do MARPSH possibilita a criação das cinco faixas de classificação de risco, segundo a pontuação alcançada: baixo, médio baixo, médio, médio alto e alto risco ${ }^{17}$.

A partir das avaliações sanitárias, os serviços de hemoterapia foram classificados de acordo com o risco potencial obtido da aplicação do MARPSH, sendo os roteiros de inspeção e as classificações de risco disponiveis no SHTWEB ${ }^{15}$.

Foram utilizadas ferramentas de estatística descritiva (gráficos e tabelas) para a organização e apresentação dos resultados. Este estudo foi aprovado pelo Comitê de Ética em Pesquisa da Secretaria Estadual de Saúde do Paraná, de acordo com o parecer 1.752.596.

\section{RESULTADOS}

A Tabela 1 descreve o total de inspeções e avaliações de risco realizadas no período de 2010 a 2017 nos serviços de hemoterapia do estado do Paraná. A tabela apresenta um total de 134 serviços no ano de 2017. Este número se refere a 104 AT, somadas a 30 serviços de hemoterapia. No estado do Paraná, ainda que uma AT esteja localizada em área intra-hospitalar, o SHTWEB contabiliza separadamente no total de serviços de saúde a agência e o serviço em que ela se localiza. 
Tabela 1 - Total de inspeções e avaliações de risco realizadas no período de 2010 a 2017

\begin{tabular}{|c|c|c|c|c|c|c|}
\hline \multirow{2}{*}{ Ano } & \multirow{2}{*}{$\begin{array}{l}\text { Total de } \\
\text { serviços }\end{array}$} & \multirow{2}{*}{$\begin{array}{c}\text { Inspecionados } \\
\text { n (\%) }\end{array}$} & \multirow{2}{*}{$\begin{array}{c}\text { Não } \\
\text { inspecionados } \\
n(\%)\end{array}$} & \multicolumn{3}{|c|}{ Avaliações de risco realizadas } \\
\hline & & & & Total & $\begin{array}{l}\text { Bancos de } \\
\text { sangue }\end{array}$ & AT \\
\hline 2010 & 126 & $72(57,1)$ & $54(42,9)$ & 55 & 21 & 34 \\
\hline 2011 & 126 & $107(84,9)$ & $19(15,1)$ & 60 & 25 & 35 \\
\hline 2012 & 128 & $107(83,6)$ & $21(16,4)$ & 41 & 14 & 27 \\
\hline 2013 & 129 & $102(79,1)$ & $27(20,9)$ & 54 & 22 & 32 \\
\hline 2014 & 131 & $93(71,0)$ & $38(29,0)$ & 55 & 18 & 37 \\
\hline 2015 & 130 & $100(76.9)$ & $30(23,1)$ & 68 & 24 & 44 \\
\hline 2016 & 130 & $101(77,7)$ & $29(22,3)$ & 68 & 24 & 44 \\
\hline 2017 & 134 & $80(59,7)$ & $54(40,3)$ & 58 & 16 & 42 \\
\hline
\end{tabular}

Legenda: $n$ - número absoluto; \% - percentagem; AT - agências transfusionais.

Fonte: Elaborada pelos autores (2017).

A Figura 1 descreve a classificação dos serviços de hemoterapia inspecionados (bancos de sangue e AT), de acordo com o risco potencial obtido da aplicação do MARPSH, no período de 2010 a 2017. Observa-se um substancial aumento das classificações de "baixo risco" a partir de 2013, sendo os percentuais de serviços classificados como "médio alto risco" e "alto risco" são relativamente baixos em todo o período.

Figura 1 - Classificação dos serviços de hemoterapia inspecionados, de acordo com o risco potencial obtido da aplicação do MARPSH (2010 a 2017).

2010

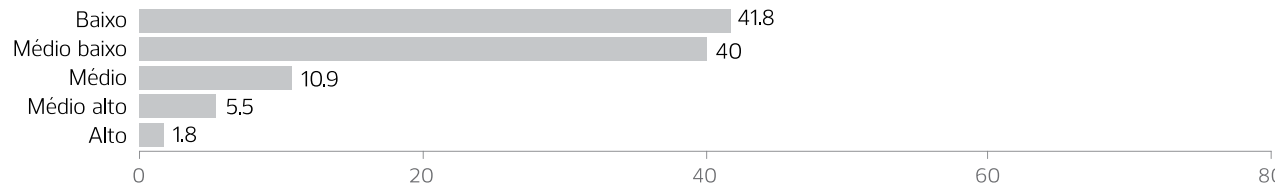

2011

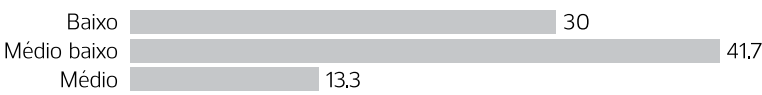

Médio alto $\quad 6.7$

Alto $\quad 8.3$

\begin{tabular}{lllll}
\hline 0 & 1 & 60 & 80
\end{tabular}

2012

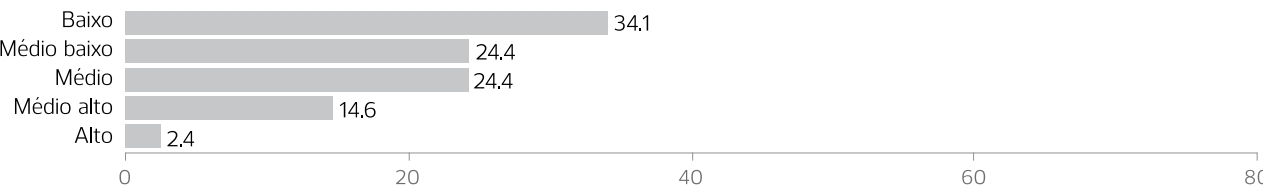




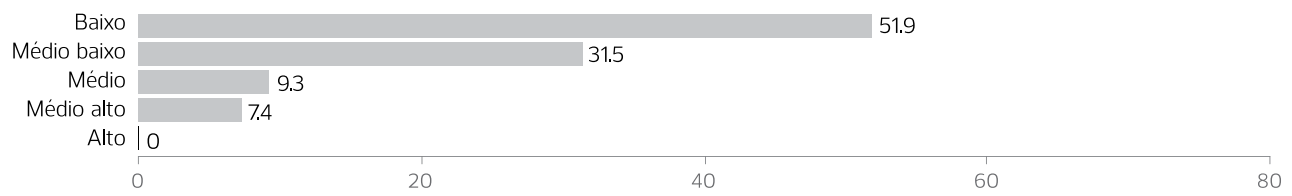

2014

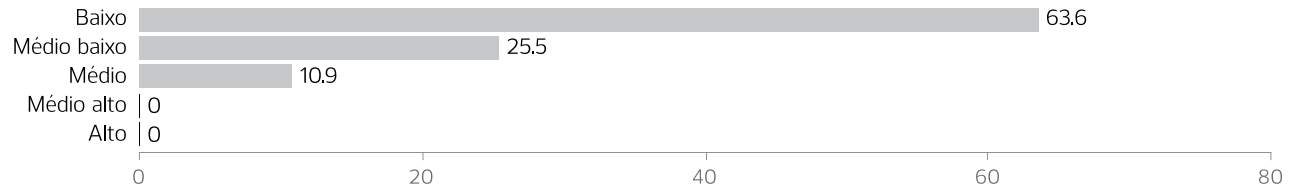

2015

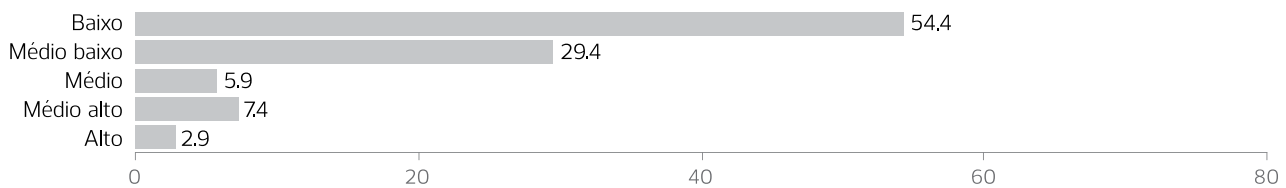

2016

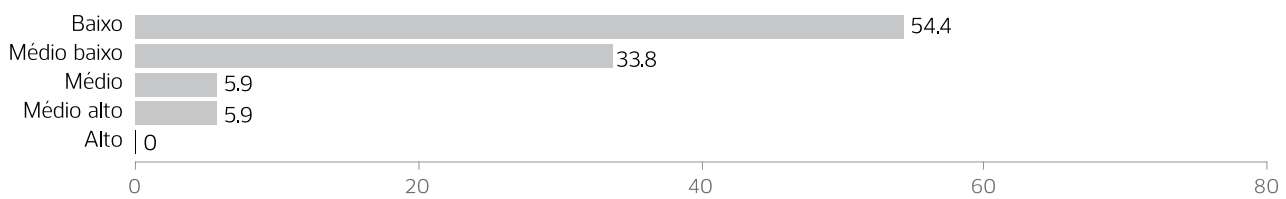

2017

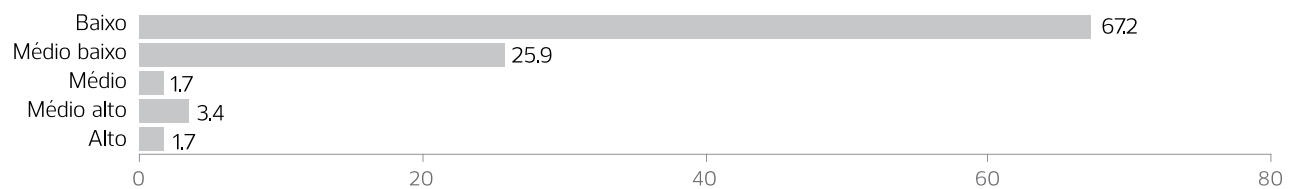

Fonte: Elaborada pelos autores (2017).

Dentre as 42 AT que passaram por avaliação de risco em 2017 (Tabela 1), 28 foram classificadas como de "baixo risco", 10 como de "médio baixo risco", uma como de "médio risco", duas como de "médio alto risco" e uma como de "alto risco". Entretanto, observou-se que 35 AT usaram a versão do MARPSH atualizada naquele ano, enquanto 7 AT usaram uma versão mais antiga. Isto impediu uma comparação da avaliação incluindo todas as AT. Assim, a Tabela 2 exibe os 10 itens com maior percentual de não conformidades, considerando as 35 AT que usaram a versão corrente do MARPSH.

Observa-se que estes itens estão concentrados nos Módulos I e V. Verifica-se, ainda que, 30\% das não conformidades são relativas à categoria Equipamentos e Dispositivos, pertencentes ao Módulo I, seguidas de 20\% na categoria Gestão da Qualidade (Módulo I). Os demais itens estão relacionados a Recursos Humanos, com um percentual de 10\% (Módulo I). Hemovigilância e Retrovigilância (10\%), Biossegurança (10\%), Infraestrutura da AT (10\%) e Procedimentos Gerais (10\%). Verificou-se que 70\% dos itens dessas não conformidades são de nível crítico II, 20\% de nível crítico III e 10\% de nível crítico I.

A Tabela 3 exibe os 10 itens com maior percentual de não conformidades, considerando as 7 AT que usaram a versão anterior do MARPSH. Observa-se que novamente a maioria dos itens pertencem ao Módulo I, seguido do Módulo V. O item mais frequente foi o "6.1. Dados Informatizados", que possui grau crítico III. 
Tabela 2 - Itens não conformes mais comuns observados nas 35 agências transfusionais, no ano de 2017, que utilizaram a versão corrente do MARPSH.

\begin{tabular}{|c|c|c|c|c|}
\hline $\begin{array}{l}\text { Código } \\
\text { MARPSH }\end{array}$ & Item avaliado & $\begin{array}{c}\text { Itens em não } \\
\text { conformidade } \\
\text { n/nt (\%) }\end{array}$ & $\begin{array}{l}\text { Não } \\
\text { se } \\
\text { aplica }\end{array}$ & $\begin{array}{l}\text { Módulo e } \\
\text { categoria }\end{array}$ \\
\hline
\end{tabular}

Documento formal (contrato

ou similar) que defina

10.2.2. responsabilidades no processo de investigação entre o fornecedor $\quad 10 / 16$ (62,5\%) $19 \quad$ Hemovigilância de hemocomponentes e o serviço transfusional

Programa de Capacitação

2.2.1. de Recursos Humanos com acompanhamento e avaliação

$\begin{array}{lll}12 / 35(34,3 \%) & 0 & \text { Recursos } \\ \text { humanos }\end{array}$

11.4.

Auditoria interna

$10 / 33(30,3 \%)$

2

Gestão da qualidade

Treinamento sistemático de pessoal

11.6.1. para toda e qualquer alteração de atividade

9/35 (25.7\%) $\quad 0 \quad$ Gestão da qualidade

Realização/registro da qualificação dos equipamentos

8/35 (22,9\%) $\quad 0 \quad \begin{gathered}\text { Equipamentos e } \\ \text { dispositivos }\end{gathered}$

Realização/registro da manutenção

7.5 corretiva e preventiva dos equipamentos

8/35 (22,9\%) $0 \quad$ Equipamentos e
dispositivos

7.5.1.

\section{Contrato e cronograma de} manutenção preventiva dos equipamentos

8/35 (22,9\%) $\quad 0 \quad \begin{gathered}\text { Equipamentos e } \\ \text { dispositivos }\end{gathered}$

Treinamento periódico da equipe envolvida em procedimentos técnicos em biossegurança,

$8 / 35(22,9 \%)$

O Biossegurança inclusive da equipe terceirizada

3.2.

Equipamentos qualificados e em conformidade com as técnicas utilizadas

$\begin{array}{ccc}8 / 35(22,9 \%) & 0 & \text { Infraestrutura } \\ \text { da AT }\end{array}$

4.5. Registros das atividades do Comitê Transfusional

$8 / 35(22,9 \%)$

Procedimentos gerais

Legenda: $n$ - número absoluto de itens em não conformidade; $n t$ - número absoluto do total de itens avaliados; \% - percentagem dos itens em não conformidade; AT - agências transfusionais. Fonte: Elaborada pelos autores (2017). 
Tabela 3 - Itens não conformes mais comuns observados nas 7 agências transfusionais, no ano de 2017, que utilizaram a versão antiga do MARPSH.

\begin{tabular}{|c|c|c|c|c|}
\hline $\begin{array}{l}\text { Código } \\
\text { MARPSH }\end{array}$ & Item avaliado & $\begin{array}{l}\text { Itens em não } \\
\text { conformidade } \\
\text { n/nt (\%) }\end{array}$ & $\begin{array}{l}\text { Não se } \\
\text { aplica }\end{array}$ & Módulo e categoria \\
\hline 6.1. & Dados informatizados & $3 / 6(50,0 \%)$ & 1 & Registros \\
\hline 3.2.3. & $\begin{array}{l}\text { Programa de Capacitação } \\
\text { de Recursos Humanos com } \\
\text { acompanhamento e avaliação }\end{array}$ & $2 / 7(28,6 \%)$ & 0 & Recursos humanos \\
\hline 8.6. & $\begin{array}{c}\text { Realização/registro da } \\
\text { qualificação dos equipamentos }\end{array}$ & $2 / 7(28,6 \%)$ & 0 & $\begin{array}{l}\text { Equipamentos e } \\
\text { dispositivos }\end{array}$ \\
\hline 11.3. & Auditoria interna & $2 / 7(28,6 \%)$ & 0 & Gestão da qualidade \\
\hline 3.1.5.1. & $\begin{array}{c}\text { Adoção e registro de medidas } \\
\text { corretivas quando identificadas } \\
\text { não conformidades }\end{array}$ & $2 / 7(28,6 \%)$ & 0 & $\begin{array}{l}\text { Procedimentos pré- } \\
\text { transfusionais }\end{array}$ \\
\hline 3.2.5. & $\begin{array}{c}\text { Programa de controle médico de } \\
\text { saúde ocupacional }\end{array}$ & $2 / 7(28.6 \%)$ & 0 & Recursos humanos \\
\hline 2.4.2. & $\begin{array}{l}\text { Controle de qualidade de cada } \\
\text { lote de reagente em uso }\end{array}$ & $2 / 7(28,6 \%)$ & 0 & Infraestrutura \\
\hline 3.1.3. & $\begin{array}{c}\text { Realização/registro de CQI } \\
\text { (controle de qualidade interno) }\end{array}$ & $2 / 7(28,6 \%)$ & 0 & $\begin{array}{l}\text { Procedimentos pré- } \\
\text { transfusionais }\end{array}$ \\
\hline 3.1.6.1. & $\begin{array}{l}\text { Reportagem ao Comitê } \\
\text { Transfusional de casos de } \\
\text { transfusões não usuais }\end{array}$ & $2 / 7(28,6 \%)$ & 0 & $\begin{array}{l}\text { Procedimentos pré- } \\
\text { transfusionais }\end{array}$ \\
\hline 3.1.1. & Médico responsável técnico & $1 / 7(14,3 \%)$ & 0 & Recursos humanos \\
\hline
\end{tabular}

Legenda: $n$ - número absoluto de itens em não conformidade; nt - número absoluto do total de itens avaliados; \% - percentagem dos itens em não conformidade.. Fonte: Elaborada pelos autores (2017).

\section{DISCUSSÃo}

Um estudo conduzido por pesquisadores brasileiros ${ }^{3}$ discutiu a importância da realização de avaliações dos Sistemas de Vigilância em Saúde Pública existentes e da análise de seus desempenhos e resultados. Tais avaliações têm a finalidade de embasar as ações de planejamento e gestão, com vistas a diminuir e prevenir riscos aos produtos e serviços ofertados e, consequentemente, melhorar a qualidade da saúde pública do País.

No presente estudo, ao descrever o universo dos serviços de hemoterapia do estado do Paraná, observou-se que, apesar de um acréscimo no número total de inspeções realizadas desde o ano de 2010 , ainda se encontra abaixo do esperado, considerando que os serviços desenvolvem em suas rotinas atividades de alta complexidade. Assim, em uma situação ideal, haveria necessidade de inspeções sanitárias em todas as unidades ativas.

Ao descrever o percentual de inspeções realizadas ano a ano, percebe-se que o número de inspeções 
sanitárias realizadas ultrapassou 80\% nos anos de 2011 e 2012 (Tabela 1). Acredita-se que tal incremento se deve às capacitações oferecidas aos técnicos de vigilância sanitária nesse período.

Verifica-se que, mesmo com a presença de um leve declínio no número de inspeções nos anos subsequentes, o percentual observado no ano de 2016 permaneceu superior ao alcançado no ano de 2010. Entretanto, no ano de 2017 houve uma acentuada queda no número de inspeções em relação a 2016. passando de 77,7\% para 59,7\%. Neste período, o Estado passou por reestruturações e reorganização financeira, influenciando o processo de trabalho de muitos segmentos em geral, e levando o número de estabelecimentos acompanhados a ser praticamente o mesmo de 2010 (Tabela 1).

Em relação às não conformidades encontradas, o fato dos serviços não utilizarem a mesma versão do MARPSH trouxe dificuldades para uma descrição da situação de riscos potenciais em que se encontram. Entretanto, em síntese, percebe-se que as não conformidades mais frequentes se relacionam a equipamentos e dispositivos, questões de infraestrutura, itens referentes à gestão da qualidade, recursos humanos e retrovigilância (Tabelas 2 e 3). Para garantir maior segurança em todo o processo transfusional, é necessário assegurar que as normas e os procedimentos sejam apropriadamente executados, e que os equipamentos e materiais funcionem corretamente, conforme disposto na Resolução RDC nº 153, de 14 de junho de 2004. da ANVISA ${ }^{17}$.

Já os itens referentes à gestão da qualidade, recursos humanos e retrovigilância são associados a questões organizacionais e relativas a processo de trabalho, e interferem diretamente na qualidade e segurança dos serviços prestados, em maior ou menor grau de criticidade ${ }^{18}$. Da mesma forma, os itens relacionados ao sistema informatizado são de extrema importância, pois garantem a rastreabilidade e a segurança das informações ${ }^{19}$. A importância de um sistema informatizado de controle de sangue e hemoderivados já era discutida em um estudo publicado em $1994^{20}$, que mostrou a utilidade deste recurso em auxiliar a adequação dos serviços às normas técnicas de procedimentos do Ministério da Saúde e, consequentemente, buscar uma melhor estrutura física e condições de atendimentos aos pacientes e doadores.

O programa de capacitação de recursos humanos, que aparece nas Tabelas 2 e 3, foi também essencial, pois trata da qualificação dos profissionais envolvidos no processo, sendo o profissional um elemento principal para a garantia da qualidade de procedimentos e processos executados em toda a cadeia transfusional' ${ }^{16}$. Alguns estudos vêm apontando lacunas importantes na formação de profissionais de hemoterapia e mostram a necessidade de revisões na formação destes profissionais, bem como programas de capacitação sobre medicina transfusional oferecidos pelas instituições ${ }^{19.21}$.

O item "auditoria" tem nível de criticidade I e foi o mais frequente entre as não conformidades. A qualificação de equipamentos, assim como cronograma de manutenção corretiva e preventiva também foram elencados dentre as não conformidades. Estes itens interferem diretamente na qualidade dos serviços prestados. Embora o item "auditoria interna" tenha peso 1 e, portanto, não confere alto risco à segurança e qualidade, quando comparado aos demais, refere-se a um item que contribui para a melhoria da qualidade dos procedimentos e processos realizados ${ }^{16}$.

Destaca-se que subnotificações e a ausência de inspeção em alguns serviços podem comprometer a extrapolação dos resultados para todo o estado. Uma potencial limitação do uso do MARPSH descrita pela literatura ${ }^{22}$ é que a complexidade dos problemas que envolvem produtos e processos dos serviços de hemoterapia dificulta o desenvolvimento de métodos que abrangem todos os aspectos relacionados ao risco.

Assim, a classificação atribuída ao serviço avaliado descreve a possibilidade de ocorrência de agravos e a fragilidade dos sistemas de controle utilizados, tornando-a insuficiente para avaliar riscos reais. Embora o 
MARPSH seja recomendado pela ANVISA como componente das boas práticas no ciclo do sangue e utilizado pelos serviços de hemoterapia ${ }^{14}$, são encontrados na literatura poucos estudos sobre os resultados de sua aplicação ${ }^{16.22}$, o que limita a comparação dos resultados encontrados neste estudo com outros obtidos em diferentes locais.

\section{CONCLUSÃO}

A presente pesquisa permitiu demonstrar a importância e a utilidade do MARPSH para as ações de inspeção sanitária. O instrumento é adotado pela vigilância sanitária no seu processo de gerenciamento de riscos em sangue no Brasil, sistematizando a verificação dos cumprimentos dos requisitos estipulados pela legislação vigente.

As limitações deste estudo incluem a possibilidade de viés de aferição dos dados, considerando ainda a chance das informações obtidas das inspeções não serem devidamente padronizadas.

Apesar destas limitações, os resultados obtidos permitem recomendar que algumas medidas sejam adotadas para a melhoria dos resultados dos serviços de hemoterapia avaliados, a saber, as prioridades relacionadas aos sistemas de informação, equipamentos e dispositivos, gestão da qualidade, recursos humanos e hemovigilância, além da necessidade de aumentar a cobertura das inspeções sanitárias com atenção àquelas classificadas como sendo de maior risco e àquelas que há algum tempo não foram inspecionadas. Ademais, são necessárias capacitações e treinamento em serviço (teórico/prático) com as equipes de vigilância sanitária.

Outra medida importante, refere-se à maior integração entre os atores do Sistema Nacional de Vigilância Sanitária, em que se inclui a solicitação de apoio e deslocamento de técnicos de diferentes regionais para participação de ação conjunta em inspeções a outros serviços que não pertencem à sua área de abrangência. Isso faz com que os técnicos conheçam outras realidades e troquem experiências entre si.

Conclui-se que os serviços avaliados necessitam se adequar aos itens não conformes por meio de medidas resolutivas que promovam maior segurança a doadores e receptores de sangue e, consequentemente. a redução dos riscos e o aumento da segurança transfusional. Para tanto, devem buscar apoio junto ao Hemocentro Coordenador na orientação quanto à adoção e implementação de medidas preventivas e corretivas.

Todas as ações devem ter um direcionamento técnico com a finalidade de otimizar recursos e ter resultados efetivos na redução do risco nos serviços de hemoterapia. Em adição, merece atenção a sensibilização dos gestores de saúde quanto às necessidades que estes serviços realmente demandam, por serem de alta complexidade e representarem serviços fundamentais de assistência à população.

\section{REFERÊNCIAS}

1. Pereima RSMR, Reibnitz KS, Martini JG, Nitschke RG. Blood donation: mechanic solidarity versus organic solidarity. Rev Bras Enferm. 2010:63(2):322-327. DOI: 10.1590/S0034-71672010000200024

2. Gurgel JLM, Carmo BBT. Dimensionamento do estoque de derivados de sangue em um hemocentro do Brasil baseado em um modelo 
de gestão de estoques e previsão de demanda. Rev Prod On-line. 2014;14(1):264-293. DOI: 10.14488/1676-1901.v14.11.1594

3. Schmunis GA, Cruz JR. Safety of the blood supply in Latin America. Clin Microbiol Rev. 2005:18(1):12-29.DOI: 10.1128/CMR.18.1.1229.2005

4. Seta MH, Silva JAA. A gestão da vigilância sanitária. In: Seta MH, Pepe VLE, Oliveira GO, organizadores. Gestão e vigilância sanitária: modos atuais do pensar e fazer. Rio de Janeiro: Editora Fiocruz, 195-217. 2006

5. Lucchese G. Globalização e regulação sanitária: os rumos da vigilância sanitária no Brasil. Brasília: Anvisa. 2008.

6. Mota DM, Freitas DRC. Araújo WN. Evaluation of the System of Sanitary Vigilance of Blood at the federal level, Brazil, 2007. Ciênc. Saúde Coletiva. 2012:17(1):191-202.doi: 10.1590/S1413-81232012000100021

7. Ministério da Saúde (BR). Resolução RDC da ANVISA n 34, de 11 de junho de 2014. Dispõe sobre as Boas Práticas no Ciclo do Sangue. Diário Oficial da União, Brasília, p. 50, 16 jun. 2014. Seção 1.

8. Ministério da Saúde (BR). Portaria de Consolidação n 5, de 28 de setembro de 2017. Consolidação das normas sobre as ações e os serviços de saúde do Sistema Único de Saúde. Diário Oficial da União, Brasília. 3out. 2017. Seção Suplemento.

9. Ministério da Saúde (BR). Portaria Conjunta ANVISA/SAS n 370, de 7 de maio de 2014. Dispõe sobre regulamento técnico-sanitário para o transporte de sangue e componentes. Diário Oficial da União, Brasília, p. 47, 8 maio 2014. Seção 1.

10. Ministério da Saúde (BR). Resolução RDC da ANVISA n 20, de 10 de abril de 2014. Dispõe sobre regulamento sanitário para o transporte de material biológico humano. Diário Oficial da União, Brasília. p. 67, 11 abr. 2014. Seção 1.

11. Ministério da Saúde (BR). Resolução RDC da ANVISA n 151, de 21 de agosto de 2001. Aprova o Regulamento Técnico sobre níveis de complexidade dos serviços de hemoterapia. Diário Oficial da União, Brasília, 22 ago. 2001. Seção 1.

12. Ministério da Saúde (BR). Resolução RDC da ANVISA n 57, de 16 de dezembro de 2010. Determina o Regulamento Sanitário para Serviços que desenvolvem atividades relacionadas ao ciclo produtivo do sangue humano e componentes e procedimentos transfusionais Diário Oficial da União, Brasília, p. 119, 17 dez. 2010. Seção 1.

13. Cunha CL. Informatização do processo de avaliação de risco sanitário em serviços de hemoterapia. [dissertação]. Florianópolis (SC): Universidade Federal de Santa Catarina; 2018

14. Silva-Junior JB, Rattner D. Segurança Transfusional: um método de Vigilância Sanitária para avaliação de riscos potenciais em serviços de hemoterapia. VigSanit Debate. 2014; 2(2): 43-52.doi: 10.3395/vd.v2i2.126

15. Secretaria de Estado da Saúde do Paraná. Resolução SESA nº 0043/2010. Dispõe sobre as condições para a instalação e funcionamento do SHTWEB - Entrada de Dados online, no Sistema Estadual de Informação e Controle Hemoterápico do Paraná - SHT e dá outras providências. [internet]. Disponivel em: http://www.saude.pr.gov.br/arquivos/File/Resolucoes2011/Resolucao0432010.pdf

16. Silva Júnior JB, Rattner D. A Vigilância Sanitária no controle de riscos potenciais em serviços de hemoterapia no Brasil. Saúde Debate. 2016:40:136-53. DOI: 10.1590/0103-1104201610911

17. Ministério da Saúde (BR). Resolução RDC da ANVISA n 153, de 14 de junho de 2004. Determina o Regulamento Técnico para os procedimentos hemoterápicos, incluindo a coleta, o processamento, a testagem, o armazenamento, o transporte, o controle de qualidade e o uso humano de sangue, e seus componentes, obtidos do sangue venoso, do cordão umbilical, da placenta e da medula óssea. Diário Oficial da União, Brasília, 24 jun. 2004. Seção 1.

18. Medeiros, AVC. Gestão da qualidade nos serviços de hemoterapia do interior do estado do Rio Grande do Norte. [Dissertação] Natal(RN): Universidade Federal do Rio Grande do Norte, Centro de Ciências da Saúde. 2018.

19. Ferreira O, Martinez EZ, Mota CA, Silva AM. Avaliação do conhecimento sobre hemoterapia e segurança transfusional de profissionais de enfermagem. Rev Bras Hematol Hemoter. 2007:29(2):160-167. DOI: 10.1590/S1516-84842007000200015

20. Pereira, SR. Avaliação preliminar do sistema informatizado de controle de sangue e hemoderivados - 1994. [Trabalho de Conclusão de Curso]. João Pessoa (PB): Centro de Pesquisas Aggeu Magalhães, Fundação Oswaldo Cruz, 1995.

21. Silva KF. Soares S, Iwamoto HH. A prática transfusional e a formação dos profissionais de saúde. Rev Bras Hematol Hemoter. 2009:31(6):421-426. DOI: 10.1590/S1516-84842009005000092

22. Silva Júnior JB, Rattner D, Martins RDCA. Controle de riscos potenciais em serviços de hemoterapia no Brasil: uma abordagem para autoridades reguladoras. Rev Panam Salud Publ. 2016:40,1-8.

RECEBIDO: $27 / 03 / 2020$

ACEITO: 09/06/2020 